Primary outcome was the score on a decision self-efficacy scale after the decision-making consultation with the oncologist.

Results Inclusion ends on 1 January 2019. Results are not yet available but we will present the first results during the conference.

Conclusion The OPTion-study provides information about the effect of a consultation, about preferred treatment goals, between GPs and older patients with non-curable cancer on self-efficacy.

\section{P62 THE DISTRESS THERMOMETER AS A PROGNOSTIC TOOL FOR ONE-YEAR SURVIVAL AMONG PATIENTS WITH LUNG CANCER}

${ }^{1} \mathrm{O}$ Geerse $^{*},{ }^{1} \mathrm{D}$ Brandenbarg, ${ }^{2} \mathrm{H}$ Kerstjens, ${ }^{1 \mathrm{~J}}$ Hoekstra-Weebers, 'S Duijts, ${ }^{1} \mathrm{H}$ Burger, ${ }^{3} \mathrm{G}$ Holtman, ${ }^{1} \mathrm{~A}$ Berendsen, ${ }^{1} \mathrm{~T}$ Hiltermann. 'University Medical Center Groningen, Groningen, Netherlands; ${ }^{2}$ University Medical Centre Groningen, GRONINGEN, Netherlands; ${ }^{3}$ University of Groningen, Faculty of Medical Sciences, Groningen, The Netherlands

\subsection{6/spcare-2019-ACPICONGRESSABS.144}

Introduction Use of patient-reported outcome measures is advocated to support high-quality cancer care. We investigated the added value of the Distress Thermometer (DT) when combined with known predictors to assess one-year survival in patients with lung cancer.

Methods Patients had newly diagnosed or recurrent lung cancer, started systemic treatment, and participated in the intervention arm of a previously published randomised trial. A Cox proportional hazards model was fitted based on five selected known predictors for survival. The DT-score was added to this model and contrasted to models including the EORTC-QLQ-C30 global QoL score or the HADS total score. Model performance was evaluated through improvement in the -2 log likelihood, Harrell's C-statistic, and a risk classification.

Results In total, 110 patients were included in the analysis of whom 97 patients accurately completed the DT. Patients with a DT score ${ }^{35}(\mathrm{~N}=51)$ had a lower QoL, more symptoms of anxiety and depression, and a shorter median survival time (7.6 months vs 10.0 months; $\mathrm{P}=0.02$ ) than patients with a DT score $<5(\mathrm{~N}=46)$. Addition of the DT resulted in a significant improvement in the accuracy of the model to predict one-year survival $(\mathrm{P}<0.001)$ and the discriminatory value $(\mathrm{C}$ statistic) marginally improved from 0.69 to 0.71 . The proportion of patients correctly classified as high risk ( ${ }^{3} 85 \%$ risk of dying within one year) increased from $8 \%$ to $28 \%$.

Conclusions Use of the DT allows clinicians to better identify patients with lung cancer at risk for poor survival, further explore sources of distress, and personalize care accordingly.

\section{P63 RECRUITING PATIENTS IN DIFFICULT ENVIRONMENTS - PRACTICAL EXPERIENCES FROM A GERMAN CACP TRIAL}

J Koch*, C PD Dr Seifart, P Von Blanckenburg. Philipps-University Marburg, MARBURG, Germany

\subsection{6/spcare-2019-ACPICONGRESSABS. 145}

Background Researchers recruiting patients for clinical - nonpharmacological - studies in palliative cancer settings face multiple problems. Patients life expectancy is limited, physical and psychological wellbeing can be volatile and medical treatment tends to be challenging and tiring for patients. Rejection and dropout rates for scientific trials in general are therefore high, especially if concerning advance care planning (ACP).

Aim Recruitment is often assigned to few members of staff who then gain a lot of experience in the matter, formal transition of knowledge can be difficult if

- a.) theoretical framework about recruitment; and

- b.) practical examples how to handle difficult recruitment conversations; are missing.

To address and overcome b.), we have developed "miniinterventions". These aim to change perspective of patients on participation in our randomized controlled ACP trial, and can be applied generally.

Results We developed several interventions covering the following topics:

-Misconceptions towards "psychological" support and academic research

-Physical and emotional wellbeing and therefore no need to partake in the trial

-Time constraints, unwillingness to invest more time in "treatment" than necessary

-Fear of burdening care givers with psychological involvement

With these "mini interventions" delegation of recruitment and training of recruitment staff is eased and the recruitment process and success does not rely solely on single experienced staff members.

Conclusion Interventions in recruitment conversations may have several effects, including improvement of patient decision making, increase of recruitment numbers and better transition of knowledge in research teams.

We seek to cross-link with other international groups facing similar challenges concerning recruiting.

\section{P65 DEVELOPMENT OF A PEDIATRIC ADVANCE CARE PLANNING INTERVENTION}

1J Fahner* ${ }^{2}$ J Rietjens, ${ }^{2} \mathrm{~A}$ van der Heide, ${ }^{1} \mathrm{~J}$ van Delden, ${ }^{1} \mathrm{M}$ Kars. ${ }^{1}$ University Medical Center Utrecht, Utrecht, Netherlands; ${ }^{2}$ Erasmus MC, Rotterdam, Netherlands

\subsection{6/spcare-2019-ACPICONGRESSABS. 146}

Background ACP-conversations in pediatrics seem to occur too late and infrequent. Standards to conduct ACP-conversations are lacking. This study describes the development of an evidence-based pediatric ACP-intervention.

Methods The Medical Research Council framework for development and evaluation of complex interventions was used to structure the developmental process. The process included a systematic review, expert review, a survey among pediatricians and qualitative research among parents, adolescents and health care professionals (HCPs). Behavioral theories and theories of coping with bereavement and loss underpinned the intervention.

Results ACP was seen as an ongoing communicative process, where children and their families work together with HCPs to discover, discuss and document values, preferences and goals of care. The intervention supports ACP by 1) educational materials, 2) an ACP conversation guide and 3) a training for HCPs. Educational materials prepare children and parents by clarifying the concept of ACP and providing preparation 
prompts to discover their values. The guide provides HCPs structures and wording to address the following identified ACP topics: the identity of the child, living with illness, the future, hope, fears and worries, preferences for daily life and goals of care. The training educates HCPs about the concept of ACP and coping with illness and loss and trains specific communication skills. The intervention includes a documentation format.

Conclusion A pediatric ACP-intervention was designed targeted to the following needs: education about the concept of ACP, strategies to conduct ACP conversations and a documentation format. Our ongoing research will evaluate the feasibility of the intervention.

\section{P66 PEDIATRICIAN'S EXPERIENCES WITH ADVANCE CARE PLANNING: A QUALITATIVE STUDY}

${ }^{1} \mathrm{~J}$ Fahner*, ${ }^{1} \mathrm{~K}$ Prinsze, ${ }^{2} \mathrm{~J}$ Rietjens, ${ }^{2} \mathrm{~A}$ van der Heide, ${ }^{1} \mathrm{~J}$ van Delden, ${ }^{1} \mathrm{M}$ Kars. ${ }^{1}$ University Medical Center Utrecht, Utrecht, Netherlands; ${ }^{2}$ Erasmus MC, Rotterdam, Netherlands

10.1136/spcare-2019-ACPICONGRESSABS.147

Background Pediatricians in academic care centers are involved in high-complex care for children with life-limiting conditions. They guide families in defining goals of care and medical decision-making. Advance Care Planning (ACP) supports family-centered care and shared decision-making. This study evaluates how pediatricians integrate ACP in their daily care for children with life-limiting conditions.

Methods An interpretative qualitative study using thematic analysis was performed. Single interviews were undertaken with 17 pediatricians working in five pediatric tertiary hospitals.

Results Initiation of ACP discussions is determined by expected disease progression, technical treatment options or increasing symptoms. Pediatricians prefer to discuss ACP within a long-term relationship with a child's family. This ensures and enables them to address issues concerning challenging medical decisions and end-of-life in the right way at the right time for a specific family. Pediatricians focus in ACP discussions on future scenario's and related care options. They try to balance between 'hope' (normalizing living with illness, focus on problem solving) and 'the worst' (decline of the child's condition and an inevitable death). They aim to prepare the child and family for 'a life as normal a possible and when inevitable, a good end of life'. Few pediatricians explore the child's and family's values and perspectives.

Conclusion Pediatricians focus on prognosis and treatment options in ACP conversations. They take families by the hand throughout the disease trajectories and try to guide medical decision making in the best interest of the child. Exploration and integration of family values seems less integrated in their practice.

\section{P67 TESTING THE EFFECTIVENESS OF A FAMILY-CENTERED PEDIATRIC ADVANCE CARE PLANNING INTERVENTION: STUDY PROTOCOL FOR A RANDOMIZED CONTROLLED TRIAL}

A van Driessche* ${ }^{*}$ A de Vleminck, K Beernaert, J Cohen, L Deliens. VUB Brussel, BRUSSEL, Belgium

10.1136/spcare-2019-ACPICONGRESSABS. 148
Background A family-centered pediatric ACP intervention (FACE) was developed in the US, which demonstrated feasibility, acceptability and safety. The aim of this study is to evaluate the effectiveness of an ACP intervention, adapted from the FACE intervention, for pediatric oncology in Flanders, Belgium.

Methods A total of 93 dyads of parents and adolescents (age 10 - 18) receiving care in pediatric oncology wards will be recruited. Intervention dyads $(\mathrm{N}=46)$ will receive three weekly 60-minute sessions. Control dyads $(\mathrm{N}=46)$ will receive care as usual. Primary outcome is congruence in treatment preferences between adolescent and parent, measured by the Statement of Treatment Preference. Secondary outcomes are: quality of communication, decisional conflict for adolescents, and quality of life of adolescents and parents. Outcomes will be measured at baseline (T0) and 3 months after the intervention (T1). A process evaluation will be done by documentation of recruitment, analyzing audio recordings of the intervention, and post-trial qualitative interviews with adolescents $(n=10)$ and parents $(n=10)$ and focus groups with the involved healthcare professionals.

Discussion This will be the first pACP intervention in Europe and will provide evidence on the effectiveness of an ACP intervention in adolescents with cancer. A process evaluation will provide in-depth insight into how the pACP intervention was delivered in practice and contribute to understanding the underlying mechanisms of the intervention.

\section{P68 DEVELOPING A PEDIATRIC ACP INTERVENTION FOR ADOLESCENTS WITH CANCER IN BELGIUM}

A van Driessche* ${ }^{*}$ A de Vleminck, K Beernaert, J Cohen, L Deliens. VUB Brussel, Brussel, Belgium

\subsection{6/spcare-2019-ACPICONGRESSABS. 149}

Background Parents of adolescents with a life-limiting illness have expressed the desire to talk to their children about goals and preferences of care. Recently, a family-centered pediatric advance care planning (ACP) intervention (FACE) was developed in the US, which demonstrated feasibility, acceptability and safety. The aim of this project is to develop a pediatric ACP (pACP) intervention by translating the existing FACE intervention and adapting it to the Belgian situation for paediatric patients with cancer and their parents.

Methods First, feedback about the proposed content and process of the FACE intervention will be obtained from pediatricians $(n=4)$, psychologists $(n=4)$, parents and adolescents with cancer $(n=8)$ from four different pediatric oncology wards through semi-structured interviews. Second, four separate focus groups with the abovementioned groups will be organized. Third, experts with extensive experience in ACP with children will review the materials individually and findings will be discussed in an expert panel. Before pilot testing the intervention, cognitive testing of all questionnaires used for the outcome measurements with adolescents, their parent $(n=4)$ and physicians $(n=4)$ will be done. All retrieved information will be processed in a first version of a manual of how the intervention is delivered.

Discussion After development of the pACP intervention, effectiveness will be tested in a randomized controlled trial design. Primary objective of the trial is to assess whether the pACP intervention improves congruence in treatment preferences 